\title{
Efecto del ramoneo bovino en renovales de Nothofagus antarctica en Chubut, Argentina, en relación con la carga ganadera y la altura de la plantas
}

\author{
Cattle browsing on Nothofagus antarctica saplings in Chubut province, Argentina, \\ related to stocking rate and plant height
}

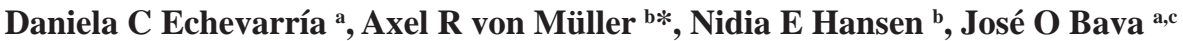 \\ ${ }^{a}$ Universidad Nacional de la Patagonia San Juan Bosco, Sede Esquel, Chubut, Argentina. \\ *Autor en correspondencia: ${ }^{\mathrm{b}}$ Instituto Nacional de Tecnología Agropecuaria, Estación Experimental Agroforestal Esquel \\ (INTA EEAf Esquel), Chubut, Argentina, vonmuller.axel@inta.gob.ar \\ c Centro de Investigación y Extensión Forestal Andino Patagónico (CIEFAP), Esquel, Chubut, Argentina.
}

\begin{abstract}
SUMMARY
In northwestern Chubut province extensive cattle rearing is the main economic activity developed under ñire canopy (Nothofagus antarctica) in silvopastoral systems with a wide range of livestock stocking rates. Cattle's browsing causes damages on ñire saplings, compromises tree regeneration and consequently woodland sustainability. In the present study, we aimed at evaluating the effects of cattle browsing on ñire tree regeneration along time, as related to stocking rate and plant height. Our study was conducted in three livestock rearing settlements in Chubut province, Argentina. Our results revealed that cattle management under low stocking rates along summer range $\left(<0.4 \mathrm{UG} \mathrm{ha}^{-1}\right.$; livestock units ha $\left.{ }^{-1}\right)$ allowed saplings apex to escape browsing with a plant minimum mean height of $1.6 \mathrm{~m}$. Moreover, under such conditions ñire saplings can tolerate domestic herbivory with compensatory growth to recover plant structures removed by cattle. Conversely, high stocking rate $\left(>0.8 \mathrm{UG} \mathrm{ha}^{-1}\right)$, for an extended period, determines suppressed tree regeneration with a shrubby architecture and lower growing rates. Under such conditions livestock exclusions are needed for at least 20 years to allow regeneration recover. It is possible to make compatible livestock production with woodland persistence by managing low cattle stocking rates and monitoring saplings height.
\end{abstract}

Key words: herbivory, regeneration, apex, damage, growth

\section{RESUMEN}

En el noroeste de la provincia de Chubut, la ganadería de cría bovina extensiva es la principal actividad productiva, y se desarrolla principalmente bajo el dosel del ñire (Nothofagus antarctica) en sistemas silvopastoriles con diferentes niveles de carga ganadera. El ganado bovino provoca daños por ramoneo sobre los renovales de ñire que compromete la regeneración y la sustentabilidad del bosque. El presente estudio evaluó el efecto del ramoneo bovino sobre la regeneración natural de ñire a lo largo del tiempo en relación a la carga ganadera y la altura de las plantas. El estudio se realizó entre los años 2005 y 2011 en tres establecimientos ganaderos de la provincia de Chubut, Argentina. Los resultados revelaron que el manejo ganadero con cargas bajas durante la veranada $\left(<0,4\right.$ unidades ganaderas ha $\left.{ }^{-1}\right)$ permite que el ápice de los renovales escape del ramoneo bovino al alcanzar una altura mínima promedio de 1,6 m. Asimismo, los renovales de ñire bajo esas condiciones toleran la herbivoría doméstica mediante mecanismos de crecimiento compensatorio de las estructuras removidas por el ramoneo bovino. Por el contrario, el manejo ganadero con cargas altas (> 0,8 unidades ganaderas ha-1) durante un período prolongado de tiempo determina una regeneración sometida por el ramoneo, con renovales bajos y arbustizados y con menores tasas de crecimiento. Bajo estas condiciones son necesarias exclusiones a la herbivoría por un período de 20 años para permitir la recuperación de la regeneración. Es posible compatibilizar producción ganadera y persistencia del bosque mediante manejo de cargas ganaderas bajas y monitoreo de la altura de la regeneración establecida.

Palabras clave: herbivoría, regeneración, ápice, daño, crecimiento.

\section{INTRODUCCIÓN}

El bosque andino patagónico del noroeste de la provincia del Chubut es la región con mayor presencia de ganado bovino de la Patagonia argentina desde mediados del siglo pasado (Guitart 2004). La zona de producción bovina es coincidente con la distribución del bosque de ñire (Nothofagus antarctica (G. Forst.) Oerst). El ñire es una especie con una amplia plasticidad ecológica en su distribución y con gran capacidad de resiliencia frente a disturbios (Donoso et al. 2006). Bajo el dosel del bosque de ñire se encuentra una amplia cantidad y diversidad de especies herbáceas de alto valor forrajero (Quinteros et al. 2010). Este ecosistema boscoso evolucionó solo con la presencia de algunos pequeños cérvidos silvestres con baja densidad (Fernández 2008) y, por lo tanto, con el advenimiento de la 
ganadería extensiva ha experimentado un incremento sustancial en la carga de herbívoros (Oesterheld et al. 1992). Estos cambios en los niveles de carga incrementan el impacto sobre el ecosistema boscoso, que puede evidenciarse en daños sobre la regeneración natural. Los elevados niveles de carga ganadera pueden comprometer la regeneración de ñire y afectar la permanencia futura de los bosques y sus funciones ecosistémicas (Bahamonde et al. 2011).

El ganado bovino, según su ecología nutricional, es un herbívoro de hábito pastoreador (Hofmann 1989). Sin embargo, cuando la cantidad de forraje herbáceo se vuelve una limitante para el consumo, ya sea por una disminución en la disponibilidad o un aumento en la presión de pastoreo, comienza a ramonear especies leñosas para compensar la deficiencia nutricional (Van Soest 1996). Este comportamiento ha sido demostrado en el bosque andino patagónico, donde la disminución en la disponibilidad de forraje se ve acompañada por un incremento en el nivel de daño sobre la regeneración natural de ñire (Hansen et al. 2008). Asimismo, Vila y Borrelli (2011) encontraron que las dietas de bovinos en el bosque andino patagónico incluían altas proporciones de Nothofagus spp., seleccionando además este género por sobre otras especies leñosas. Si bien se consideran a los bosque de ñire con gran capacidad de resiliencia frente a los disturbios (Donoso et al. 2006), el ramoneo reiterado sobre los renovales establecidos compromete la cantidad y calidad de la regeneración natural (Premoli 1991).

En general, el daño por ramoneo sobre especies leñosas produce una disminución de biomasa vegetal y de su altura, generando además, deformaciones y aumento en la cantidad de ramificaciones (Briske 1996). Sin embargo, las plantas poseen diversas adaptaciones para evitar o tolerar la herbivoría, desarrolladas a partir de la co-evolución de interacciones planta-animal (Briske 1996). Entre estas adaptaciones se encuentra el crecimiento compensatorio, un mecanismo diferido en el tiempo que permite a las plantas restituir biomasa removida por los herbívoros como respuesta ecofisiológica luego de cambios en su estructura morfológica (Belsky et al. 1993, Giorgis et al. 2010).

Las plantas que se encuentran expuestas a altas cargas ganaderas suelen ser más ramoneadas, tanto en cantidad de plantas como de ramas por planta, respecto a aquellas expuestas a cargas bajas o moderadas (Teich et al. 2005, Giorgis et al. 2010). Asimismo, el ramoneo de los herbívoros sobre el ápice determina retardo en el crecimiento, y es posible asociar mayores niveles de daño con aumentos en la carga ganadera (Marquardt et al. 2009). En estudios realizados en Patagonia con exclusión de ganado, las plantas leñosas duplicaron su tasa de crecimiento en altura respecto a las expuestas (Raffaele et al. 2007, Blackhall et al. 2008). También se observó que el daño sobre la regeneración natural está vinculado a la cercanía de ambientes abiertos y empastados (Quinteros et al. 2012). Esto demuestra la heterogeneidad espacial en los niveles de daño como consecuencia de la carga localizada, que resulta independiente de la carga ganadera general (von Müller et al. 2013a)
El tamaño de las plantas es otro aspecto relevante que influye sobre el daño que pueden provocar los herbívoros (Giorgis et al. 2010). Plantas más pequeñas, y por lo tanto más accesibles, son las que presentan mayor susceptibilidad al ramoneo en sus zonas meristemáticas (Marquardt et al. 2009). En Patagonia, se observó que el ramoneo apical de renovales de Nothofagus spp. se produce hasta 2,5 metros de altura (Peri et al. 2009). En este sentido, Blackhall et al. (2008) comprobaron que los renuevos protegidos del ganado duplican la altura máxima respecto de los expuestos.

El objetivo general del presente trabajo es evaluar el ramoneo bovino sobre la regeneración natural de ñire a lo largo del tiempo en el bosque andino patagónico del noroeste de Chubut en relación con la carga ganadera y la altura de las plantas. La hipótesis general del trabajo es que la magnitud del ramoneo sobre ñire depende de la altura de las plantas y la carga animal, pero con efectos espacialmente localizados dentro de los cuadros ganaderos. Para ello se proponen los siguientes objetivos específicos: (a) evaluar el daño apical y lateral en renuevos de ñire causado por ganado bovino en relación a la carga ganadera y a la altura de las plantas; (b) analizar la relación de la tasa de crecimiento anual con la altura de las plantas, y (c) relacionar las deformaciones producidas por ramoneo con la carga ganadera.

\section{MÉTODOS}

Área de estudio. El área de estudio se encuentra entre los departamentos Futaleufú y Languiñeo, en el noroeste de la provincia del Chubut, aproximadamente entre los $43^{\circ} 09^{\prime}$ y $43^{\circ} 35^{\prime} \mathrm{S}$, y los $71^{\circ} 15^{\prime}$ y $71^{\circ} 45^{\prime} \mathrm{O}$. El área se ubica fitogeográficamente dentro del dominio Subantártico, provincia Subantártica y el Distrito del Bosque Caducifolio, caracterizado por la presencia de especies de follaje caedizo como lenga y ñire entre las dominantes (Reque et al. 2007). El clima de la región es templado-frío húmedo cordillerano, con una temperatura media anual de $12,2{ }^{\circ} \mathrm{C}$, con valores medios de 17,4 y $3,8{ }^{\circ} \mathrm{C}$ para los meses de enero y julio respectivamente; además, no se registra período libre de heladas en ningún momento del año. El nivel de precipitaciones es entre 900 y $1.000 \mathrm{~mm}$ año-1 y ocurren principalmente en otoño e invierno (70 \%) en forma de lluvia o nieve, en tanto que, en la época primavero-estival ocurre el $30 \%$ restante. Los suelos son en general molisoles con profundidades de al menos $80 \mathrm{~cm}$ efectivos y sin limitaciones para el establecimiento forestal (Quinteros et al. 2010).

En el noroeste de Chubut, los bosques de ñire se clasifican en tres tipos forestales según su altura dominante: ñirantales bajos de 0 a 4 m; ñirantales intermedios de 4,1 a 8 m; y ñirantales altos, mayores a 8,1 $\mathrm{m}$ de altura (Hansen et al. 2004). En bosques altos y de cobertura media se desarrollan numerosas especies de gramíneas y hierbas forrajeras bajo el dosel con productividades de $1.200 \mathrm{~kg} \mathrm{ha}^{-1} \mathrm{año}^{-1} \mathrm{de}$ materia seca (Hansen et al. 2008). Se realiza un manejo silvopastoril del bosque de ñire obteniendo como princi- 
pales productos terneros y leña (Guitart 2004, Reque et al. 2007). Entre las especies leñosas del sotobosque se encuentran Schinus patagonicus (Phil.) I.M. Johnst. Ex Cabrera, Berberis microphylla G. Forst. y Ribes cucullatum Hook. et Arn.; y entre las especies herbáceas se encuentran Potentilla chiloensis (L.) Mabb., Anemone multifida Poir., Acaena pinnatifida Ruiz et Pav., Geranium sessiliflorum Cav., Taraxacum officinale F.H. Wigg. y otras especies de mayor valor forrajero como Bromus sp., Dactylis glomerata L., Trifolium repens L., Holcus lanatus L., Poa sp. y Rumex acetosella L. (Quinteros et al. 2010).

Establecimiento de categorías de carga ganadera general. El trabajo se realizó en tres establecimientos entre los años 2005 y 2011, dos de los cuales están ubicados en la localidad de Lago Rosario (4315’44,2” S; 71²5’4,8” O; 773 m s.n.m.; y $43^{\circ} 12^{\prime} 34,6^{\prime \prime}$ s; $71^{\circ} 24^{\prime} 42,4^{\prime \prime}$ O; 700 m s.n.m.), mientras que el tercero está ubicado en la localidad de Corcovado (43³0'26,2” S; 71²4’20,2” O; 712 m s.n.m.). Inicialmente, en el año 2005 los establecimientos seleccionados fueron categorizados según la carga ganadera reportada por los propietarios durante la veranada en: (A) alta carga ganadera uno de los establecimientos de Lago Rosario con aproximadamente $0,8 \mathrm{UG} \mathrm{ha}^{-1}$ [unidades ganaderas por hectárea]; y (B) baja carga ganadera a los dos establecimientos restantes con aproximadamente $0,4 \mathrm{UG} \mathrm{ha}^{-1}$. La veranada bovina se desarrolla anualmente en los predios desde el mes de diciembre hasta mayo inclusive (6 meses); por ello si se considera el descanso del resto del año (junio a noviembre), la carga media anual de los establecimientos sería aproximadamente la mitad de los valores de veranada (Hansen et al. 2014). Durante el período de estudio, el manejo ganadero así como las cargas se mantuvieron relativamente estables. Esta categorización por carga ganadera permitió realizar un estudio del impacto del ramoneo bovino a una escala predial, es decir, a nivel de establecimiento.

Diseño de muestreo. En el año 2005 se seleccionaron inicialmente para la evaluación, tres parcelas de aproximadamente una hectárea de superficie, en un sitio clasificado como de buena calidad con altura dominantes de dosel de ñire de entre 9 y 10 metros (Hansen et al. 2004, Ivancich et al. 2011). En las mismas se seleccionaron al azar 90 renovales de ñire establecidos de semilla en cada uno de los tres establecimientos ( $n=90 ; N=270)$ y categorizados según su altura. Los renovales seleccionados fueron individualizados y marcados mediante una estaca de madera numerada. Luego, en el año 2011 se realizó la remedición de los mismos individuos, identificándolos por su número en la estaca, de manera de remedir el mismo individuo que en el año 2005, si es que este estaba vivo. Sin embargo, debido a cambios en el uso del bosque se perdieron por completo dos parcelas del establecimiento, ubicado en la localidad de Corcovado, quedando sólo una parcela de las tres originales para ser reevaluada. Además, un cierto número de individuos evaluados en 2005, no pudieron ser relocalizados en 2011. Por todo eso, del año 2005 se consideraron un total de 197 renovales, mientras que en 2011 fueron reevaluados 169.

Cuantificación de cargas ganaderas localizadas. Considerando la alta heterogeneidad en la distribución del ganado bovino en cuadros ganaderos (localmente llamados "potreros") extensivos del bosque andino patagónico (von Müller et al. 2013a), en el año 2011 y conjutamente con la evaluación de renovales, se estimaron valores de carga ganadera localizada [UG ha-1] en cada una de las parcelas individualmente. La estimación se realizó de manera indirecta a través de la frecuencia de deposiciones bovinas en el suelo, realizando 100 tiros de un cuadrado de 30 por 30 cm y evaluando frecuencia de positivos con presencia efectiva de heces sobre el total de tiros (ver detalles metodológicos en von Müller et al. 2012). Esto permitió calcular una carga ganadera puntual al momento de la evaluación de los renovales. La fórmula utilizada fue: carga ganadera localizada [UG ha-1] $=0,014 \times$ frecuencia de deposiciones [\%]. Este estudio, a diferencia del estudio a escala predial, permite cuantificar cargas bovinas localizadas en la parcela, evidenciando de esta manera un efecto bovino directo de al menos los últimos dos años.

Categorización de la regeneración natural. En el año 2005 se realizó una categorización de los renovales de ñire según clases de altura por su accesibilidad diferencial al ramoneo bovino: a) renovales chicos, $\leq 0,50 \mathrm{~m}$; b) renovales medianos, de $0,51 \mathrm{a} 1,50 \mathrm{~m}$; y por último c) renovales grandes, > 1,50 m, considerándose plantas de hasta aproximadamente $3 \mathrm{~m}$ de altura (10 plantas de cada categoría por parcela). En el año 2005 se relevaron 59 renovales chicos, 68 medianos y 70 grandes, pero se trabajó como mínimo con seis renovales por categoría y por parcela. En cada planta se evaluó la altura total, el daño por ramoneo en ramas laterales, el ramoneo del ápice caulinar, la cantidad de ramificaciones, el diámetro a la altura del cuello (DAC), y solo en la categoría superior, el diámetro a la altura del pecho (DAP). En el año 2011, se reevaluaron 45 renovales chicos, 60 medianos y 64 grandes, manteniendo cada renoval la categorías definida originalmente en el año 2005, y repitiéndose las anteriores mediciones.

Tasa de mortalidad de renovales. Entre ambos muestreos se calculó la mortalidad de renovales de ñire respecto a la totalidad de los 197 individuos contemplados en 2005. La tasa de mortalidad entre ambos muestreos fue evaluada mediante ANDEVA simple, considerando como factor la categoría de altura de los renovales. Los datos fueron previamente transformados mediante raíz cuadrada para poder ser analizados mediante modelos paramétricos.

Daño en ramas laterales. Se evaluó como variable respuesta el porcentaje de ramas laterales dañadas por ramoneo bovino, respecto al total de ramas de la planta (Hansen et al. 2008). Se observó individualmente cada rama y cual- 
quier evidencia de ramoneo fue considerada como rama lateral dañada. El primer factor que se incluyó fue el año de evaluación (2005 y 2011), y considerando que se evaluaron las mismas plantas, la evaluación estadística utilizada fue un modelo lineal general de medidas repetidas. Se consideraron además otros dos factores en modelos diferentes, por un lado, la carga ganadera general (alta y baja), y, por el otro, la categoría de renovales (chicos, medianos y grandes). Además, se consideraron las interacciones entre los factores. Los datos de daño lateral en ambos modelos, por ser valores porcentuales, fueron previamente transformados mediante raíz cuadrada para poder ser analizados mediante modelos paramétricos.

Daño apical terminal. En cada ranoval se evaluó particularmente el ramoneo del ápice, considerando la variable respuesta ramoneado (1) o no ramoneado (0). La distribución binominal de los datos determinó que fueran analizados mediante regresión logística y a escala de parcela. Se realizó un modelo logístico para cada año de evaluación (2005 y 2011), y como variable explicatoria se incluyó la altura de los renovales al momento de la evaluación. Esto permitió encontrar una altura de renovales a la cual baja la probabilidad de ramoneo del ápice por parte del bovino, definida como altura de escape mínima promedio. Este punto se ubica en el punto de inflexión de la curva de regresión logística $(\mathrm{y}=0,5)$. En una regresión lineal simple posterior se vinculó la altura de escape mínima promedio de la parcela con la carga ganadera localizada con el objetivo de interpretar, según la carga ganadera, hasta que altura pueden en promedio ramonear los bovinos el ápice de ñire. El análisis se realizó con carga localizada, pero el promedio de sus valores integra la carga general del establecimiento (von Müller et al. 2012).

Altura y tasa anual de crecimiento de renovales. Se evaluó como variable respuesta la altura total de renovales en ambos años de muestreo (2005 y 2011) mediante un modelo lineal general de medidas repetidas, considerando el efecto año como primer factor. Se consideró como segundo factor la categoría de renovales (chicos, medianos y grandes) y la interacción entre ambos factores. Se realizó además una prueba pos-hoc de Bonferroni para hacer comparaciones múltiples entre alturas medias de las categorías de renovales. Por otro lado, la diferencia de altura de renovales en las cinco temporadas estivales de crecimiento consideradas en este estudio (2005-2011) permitió calcular como variable respuesta una tasa anual de crecimiento (o decrecimiento) en altura ( $\mathrm{cm}$ año $\left.{ }^{-1}\right)$ para las diferentes categorías. Las diferencias se analizaron mediante un ANDEVA paramétrico, y luego se realizó una prueba a posteriori para comparar diferencias mínimas significativas (DMS) por contrastes univariados en el factor categoría.

Diámetro a la altura del cuello y tasa anual de crecimiento. Se evaluó como variable respuesta el diámetro a la al- tura del cuello (DAC) en ambos años de muestreo (2005 y 2011) mediante un modelo de medidas repetidas, considerando el efecto año como primer factor. Se consideró como segundo factor la categoría de renovales (chicos, medianos y grandes) y la interacción entre ambos factores. Se realizó además una prueba pos-hoc de Bonferroni para hacer comparaciones múltiples entre los DAC medios de las categorías de renovales. Por otro lado, la diferencia en DAC en las cinco temporadas estivales de crecimiento consideradas en este estudio (2005-2011) permitió calcular como variable respuesta una tasa anual de crecimiento (o decrecimiento) en diámetro $(\mathrm{cm}$ año-1) para las diferentes categorías de renovales. Las diferencias se analizaron mediante un ANDEVA paramétrico, y luego se realizó un test a posteriori para comparar diferencias mínimas significativas (DMS) por contrastes univariados en el factor categoría.

Tiempo de escape del ápice al ramoneo. La relación entre altura de escape mínima promedio por categoría de altura y la tasa de crecimiento anual en altura permitió obtener un tiempo mínimo promedio, en años, que necesita el ápice de los renovales para escapar al ramoneo. Este término fue definido como tiempo de escape del ápice al ramoneo y se evaluó en cada parcela diferenciando por categoría y considerando además que las plantas al crecer cambian de categoría de altura y, por lo tanto, de tasa de crecimiento. El tiempo de escape se analizó estadísticamente mediante un modelo lineal general en el cual la parcela fue la unidad de muestreo $(\mathrm{n}=7)$. Se incluyó como factores a la categoría de altura de renovales (chicos, medianos y grandes) y la carga ganadera general (alta y baja). Asimismo, se realizó un prueba de contrastes univariados para evaluar diferencias mínimas significativas (DMS) en la categoría de altura.

Número de ramificaciones. Se evaluó la variable respuesta a la cantidad de ramificaciones presentes en los renovales de ñire en ambos años de muestreo (2005 y 2011) mediante un modelo de medidas repetidas considerando el efecto año como primer factor. Se consideraron además otros dos factores en modelos diferentes, carga ganadera general (alta y baja), y categoría de renovales (chicos, medianos y grandes). Además, se consideraron las interacciones entre factores.

Deformaciones. A partir del número de ramificaciones y la altura de los renovales de ñire se calculó para cada planta un índice de arbustización (IA), que consistió en el cociente entre la cantidad de ramas totales y su altura, similar al utilizado por Renison et al. (2005). La evaluación estadística se realizó mediante un modelo de medidas repetidas, considerando como primer factor el año (2005 y 2011). Se consideró como segundo factor la carga ganadera general del predio (alta y baja) y las interacciones entre ambos factores. 


\section{RESULTADOS}

Tasa de mortalidad de renovales. Fue posible establecer que entre ambos años de muestreo hubo una tasa de mortalidad promedio de $14 \%$. Sin embargo, la tasa fue significativamente mayor en renovales chicos (28,00 \pm 8,35 \%), respecto a medianos $(11,86 \pm 5,36 \%)$ y grandes $(8,57 \pm 4,04 \%)$ ( $\mathrm{F}=3,41 ; P=0,0414)$.

Cargas ganaderas localizadas. Las parcelas con mayores cargas ganaderas localizadas se encontraron en el establecimiento con carga general alta (A) ubicado en la localidad de Lago Rosario, siendo sus valores de: A1 = 0,748 UG ha-1 A2 $=0,806 \mathrm{UG} \mathrm{ha}^{-1} ; \mathrm{y} \mathrm{A} 3=0,864 \mathrm{UG} \mathrm{ha}^{-1}$. Los valores encontrados en los establecimientos con carga ganadera baja (B) fueron: B1 = 0,226 UG ha-1; B2 = 0,313 UG ha-1; y B3 $=0,690 \mathrm{UG} \mathrm{ha}^{-1}$ en Lago Rosario, y B4 = 0,313 UG ha-1 en Corcovado.

Daño en ramas laterales. El daño provocado por el ramoneo bovino en ramas laterales tuvo un incremento marginalmente significativo entre el año 2005 y el año 2011 ( $F=2,984 ; P=0,086$; figura $1 \mathrm{~A}$ ). Sin embargo, no hubo diferencias significativas entre los establecimientos de alta y baja carga ganadera ( $\mathrm{F}=0,016 ; P=0,899$; figura $1 \mathrm{~A}$ ). Tampoco fue posible encontrar interacciones significativas entre año y carga ganadera general, lo cual indica que las tendencias en el incremento en el daño son similares en ambos niveles de carga ( $\mathrm{F}=0,848 ; P=0,358$; cuadro 1 ).
El daño lateral tuvo un efecto significativo del año ( $\mathrm{F}=5,456 ; P=0,021$; figura $1 \mathrm{~B})$ y una interacción significativa entre año y categoría ( $\mathrm{F}=6,645 ; P=0,002$; cuadro 1). Esto pudo evidenciarse en que las categorías de renovales chicos y medianos tuvieron un incremento en el nivel de daño, en tanto que los renovales grandes tuvieron una disminución en el nivel de daño (figura 1B). El factor categoría de altura fue marginalmente significativo en el modelo ( $\mathrm{F}=2,971 ; P=0,054)$. En el año 2005 no hubo diferencias en el nivel de daño entre categorías, pero si las hubo en el año 2011 de renovales grandes respecto a las demás (figura 1B).

Daño apical terminal. En el año 2005, la regresión logística general entre la altura de la planta y el ramoneo del ápice fue significativa (Wald $C h i^{2}=4,89 ; P=0,0270$; cuadro 1 ), pero al incluir la parcela como variable de clasificación, esta no fue significativa. En el año 2011, la regresión logística general entre la altura de las plantas y el ramoneo del ápice resultó significativa (Wald $C h i^{2}=19,69 ; P \leq 0,001$; figura 2A), permitiendo calcular una altura de escape mínima promedio de los renovales para 2011 de 1,61 m. En el modelo de regresión logística fue significativa la parcela como variable de clasificación ( $P=0,0009$; figura 2A). La altura de escape mínima promedio de los renovales varió entre 47 y $253 \mathrm{~cm}$ (figura 2A). Al relacionar los valores de altura de escape mínima promedio de la parcela con su carga ganadera localizada, se encontró que en la medida que aumenta la carga localizada, el ganado bovino aumen-
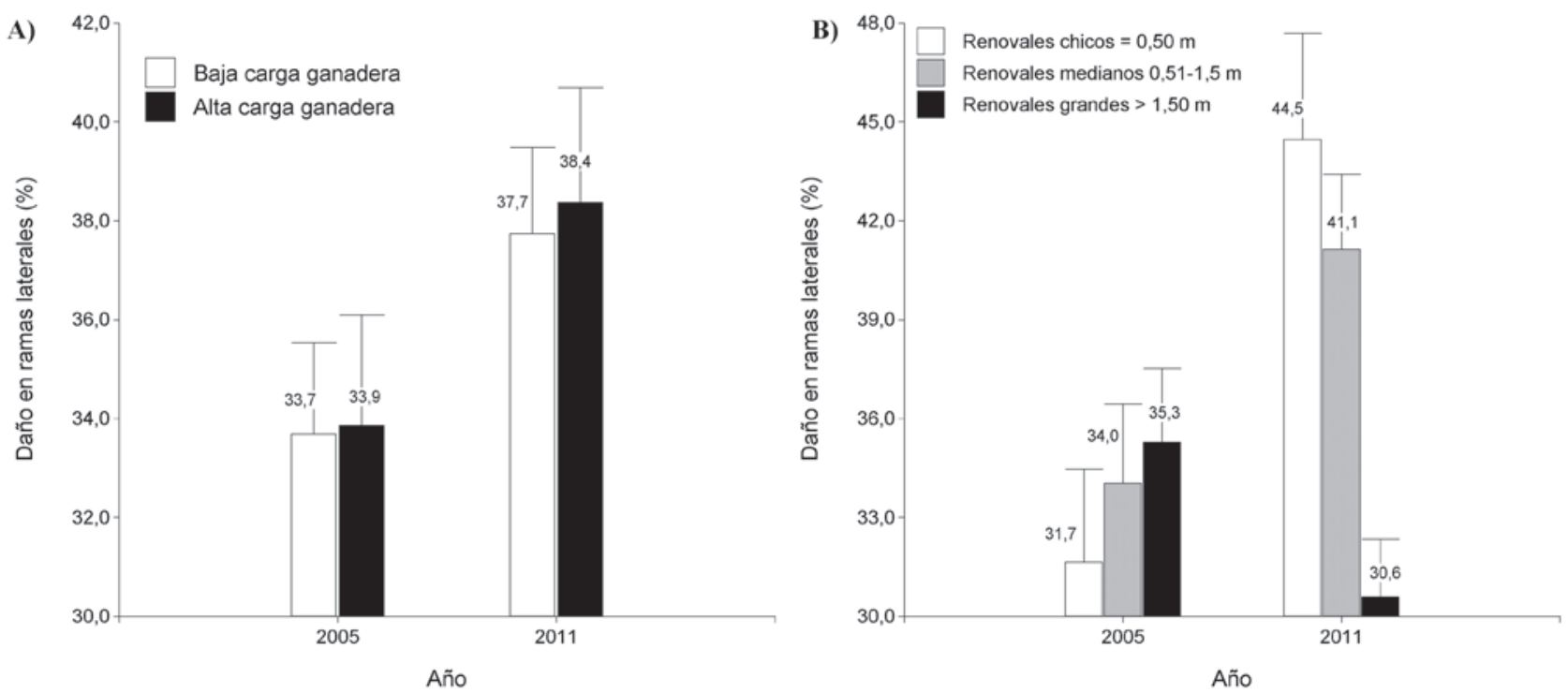

Figura 1.A) Daño medio en ramas laterales de las plantas $\left(\% \pm \mathrm{EE}^{1}\right)$ en establecimientos con baja y alta carga ganadera general para los años 2005 y 2011. B) Daño medio en ramas laterales de las plantas (\% $\pm \mathrm{EE}$ ) para las diferentes categorías de altura de renovales $(\leq 0,5 \mathrm{~m}, 0,5-1,5 \mathrm{~m}$ y $>1,5 \mathrm{~m})$ para los años 2005 y $2011 .{ }^{1}$ Error Estándar.

A) Mean values of browsing damage in lateral plant shoots $\left(\% \pm \mathrm{SE}^{2}\right)$ in low and high cattle stocking rate settlements for both sampling years 2005 and 2011. B) Mean values of browsing damage in lateral plant shoots $(\% \pm$ SE) at different plant height categories $(\leq 0.5 \mathrm{~m}, 0.5-1.5 \mathrm{~m}$ and $>1.5 \mathrm{~m}$ ) for both sampling years 2005 and $2011 .^{2}$ Standard error. 
Cuadro 1. Modelos estadísticos para analizar el ramoneo de ganado bovino sobre Nothofagus antarctica para las variables respuesta consideradas con detalles de factores, interacciones, pruebas pos-hoc y niveles de significancia.

Statistical models to analyze cattle browsing effect on Nothofagus antarctica for the different response variables with details on factors, interactions, pos-hoc tests and significance levels.

\begin{tabular}{|c|c|c|c|c|c|c|}
\hline Modelo estadístico & $\begin{array}{l}\text { Variable respuesta } \\
\text { (unidad) }\end{array}$ & Factor 1 & Factor 2 & Interacción & Pos-hoc & $\begin{array}{c}\text { Figura } \\
\text { (en Resultados) }\end{array}$ \\
\hline ANDEVA & Tasa de mortalidad (\%) & Categoría $\left.{ }^{*}\right)$ & - & - & - & - \\
\hline Medida repetida & Daño ramas laterales (\%) & Año ${ }^{(m s)}$ & Carga $^{(n s)}$ & Año*Carga ${ }^{(n s)}$ & DMS $^{1}$ & 1a \\
\hline Medida repetida & Daño ramas laterales (\%) & Año ${ }^{(*)}$ & Categoría (ms) & Año*Categoría $\left.{ }^{*}\right)$ & $\mathrm{DMS}^{1}$ & $1 b$ \\
\hline Regresión logística & Daño apical 2005 (0/1) & Altura ${ }^{(*)}$ & Parcela ${ }^{(n s)}$ & - & - & - \\
\hline Regresión logística & Daño apical 2011 (0/1) & Altura $^{(* *)}$ & Parcela ${ }^{(* *)}$ & - & - & $2 \mathrm{a}$ \\
\hline Regresión lineal & $\begin{array}{l}\text { Altura de escape mínimo } \\
\text { promedio }(\mathrm{cm})\end{array}$ & Carga Loc..$^{(* *)}$ & - & - & - & $2 b$ \\
\hline Medida repetida & Altura renovales (cm) & Año ${ }^{(* * *)}$ & Categoría $^{(*)}$ & Año*Categoría ${ }^{(* *)}$ & Bonf. ${ }^{(*)}$ & 3a \\
\hline ANDEVA & $\begin{array}{l}\text { Tasa anual de crecimiento } \\
\text { en altura }(\mathrm{cm} \text { año-1) }\end{array}$ & Categoría ${ }^{(* *)}$ & - & - & $\mathrm{DMS}^{1}$ & За \\
\hline Medida repetida & DAC de renovales $(\mathrm{cm})$ & Año ${ }^{(* *)}$ & Categoría $\left.{ }^{*}\right)$ & Año*Categoría ${ }^{(* * *)}$ & Bonf. ${ }^{(*)}$ & $3 b$ \\
\hline ANDEVA & $\begin{array}{l}\text { Tasa anual de crecimiento } \\
\text { en diámetro }\left(\mathrm{cm} \text { año }{ }^{-1}\right)\end{array}$ & Categoría ${ }^{(* *)}$ & - & - & $\mathrm{DMS}^{1}$ & $3 b$ \\
\hline Modelo lineal & $\begin{array}{l}\text { Tiempo de escape ápice } \\
\text { (años) }\end{array}$ & Categoría ${ }^{(* *)}$ & $\operatorname{Carga}^{(*)}$ & - & $\mathrm{DMS}^{1}$ & - \\
\hline Medida repetida & Número ramificaciones & Año ${ }^{(* * *)}$ & Carga ${ }^{(m s)}$ & Año*Carga ${ }^{(*)}$ & $\mathrm{DMS}^{1}$ & $4 a$ \\
\hline Medida repetida & Número ramificaciones & Año ${ }^{(* * *)}$ & Categoría ${ }^{(* * *)}$ & Año*Categoría ${ }^{(* *)}$ & $\mathrm{DMS}^{1}$ & $4 \mathrm{~b}$ \\
\hline Medida repetida & Índice arbustización & Año (ns) & $\operatorname{Carga}^{(* *)}$ & Año*Carga ${ }^{(n s)}$ & DMS $^{1}$ & 5 \\
\hline
\end{tabular}

ms marginalmente significativo $\mathrm{P} \sim 0,05 ;{ }^{*}$ significativa a $P \leq 0,05 ;{ }^{* *}$ significativa a $P \leq 0,01 ;{ }^{* * *}$ significativa a $P \leq 0,001 ;{ }^{\text {ns }}$ no significativa. ${ }^{1}$ DMS: diferencia mínima significativa por contrastes univariados.
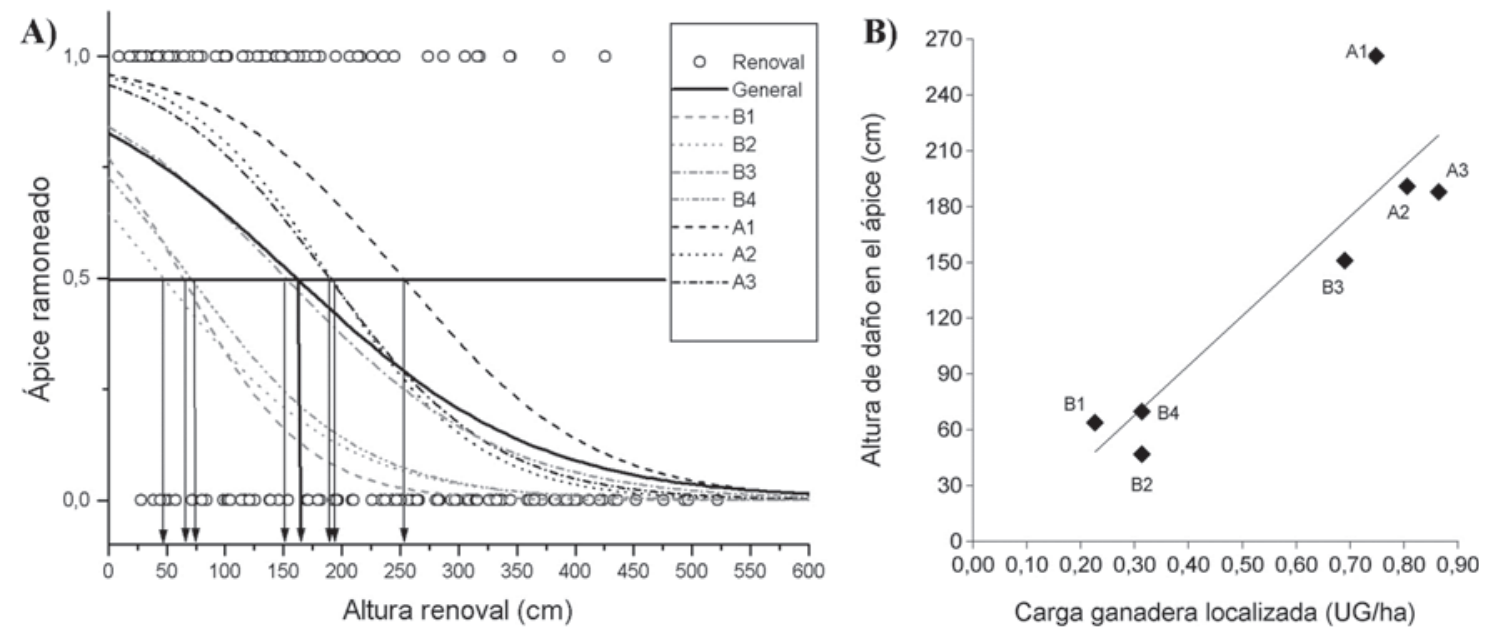

Figura 2. A) Modelo de regresión logística general (general) y para cada una de las parcelas (A1, A2, A3, M1, M2, M3 y B1) entre la altura de los renovales ( $($ ) y el ramoneo del ápice en el año 2011. Valores de $\mathrm{y}=1$ indican ápice ramoneado y valores de $\mathrm{y}=0$ indican ápice no ramoneado. El valor y = 0,5 indica el umbral de altura de la planta sobre el cual el ápice deja de estar ramoneado, y por lo tanto, define la altura de escape mínima promedio. B) Regresión lineal entre la altura de escape mínima promedio del ápice de cada parcela

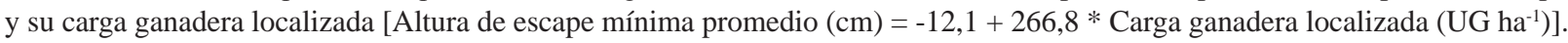

A) General logistic regression model (general) and for each plot (A1, A2, A3, B1, B2, B3 and B4) between plant height ( 0 ) and browsed shoot apex at the year 2011. Values of $y=1$ indicates a browsed shoot apex and values of $y=0$ indicates a non-browsed shoot apex. Value $y=0.5$ indicates the threshold in plant height above which the shoot apex quit browsing, thus defining mean minimum escaping height. B) Linear regression between mean minimum escaping height from the plot and its localized stocking rate [Mean minimum escaping height $(\mathrm{cm})=-12.1+266.8 *$ Localized stocking rate $\left(\mathrm{AU}^{*}\right.$ ha-1)]. *Animal Units. 
ta lineal y positivamente la altura a la cual daña el ápice de la regeneración $\left(\mathrm{R}^{2}=0,801\right.$ y $P=0,0068$; figura $\left.2 \mathrm{~B}\right)$.

Altura y tasa anual de crecimiento. Las alturas de la regeneración de ñire registradas en el año 2011 fueron significativamente mayores a las de 2005 en todas las categorías de altura de renovales definidas originalmente $(\mathrm{F}=209,81$; $P \leq 0,001$; figura $3 \mathrm{~A}$ ). Se encontraron además interacciones significativas entre los factores año y categoría de renovales ( $\mathrm{F}=16,80 ; P \leq 0,001)$, lo cual nos indicó que los cambios en las alturas de los renovales entre ambos años de muestreo no fueron iguales en cada una de ellas (figura 3A). En prueba pos-hoc de Bonferroni se encontró que las categorías de renovales tenían diferencias significativas entre sus alturas medias $(P<0,05$; cuadro 1$)$. Por otro lado, se encontró que la categoría de renovales grandes tuvieron significativamente mayor tasa anual de crecimiento en altura respecto a la categoría medianos, y esta a su vez con la categoría chicos ( $\mathrm{F}=13,35 ; P \leq 0,001$; ver conectores entre ambos años en la figura $3 \mathrm{~A}$ ).

Diámetro a la altura del cuello y tasa anual de crecimiento. Los DAC de la regeneración de ñire registradas en el año 2011 fueron significativamente mayores a las de 2005 en todas las categorías de altura de renovales definidas al inicio del estudio ( $F=345,56 ; P \leq 0,001$; figura $3 B$ ).
Se encontraron además interacciones significativas entre año y categoría de renovales ( $\mathrm{F}=38,07 ; P \leq 0,001$ ), lo cual nos indicó que los cambios en el DAC de los renovales entre ambos años de muestreo no fueron iguales en cada una de las categorías (figura 3B). En prueba pos-hoc de Bonferroni se encontró que las categorías tenían diferencias significativas entre sus DAC medios $(P \leq 0,05$; cuadro 1). Por otro lado, se encontró que la categoría de renovales grandes tiene significativamente mayor tasa anual de crecimiento en diámetro respecto a la categoría medianos, y estos a su vez con la categoría de renovales chicos ( $\mathrm{F}=38,07 ; P \leq 0,001$; ver conectores entre ambos años en la figura $3 \mathrm{~B})$.

Tiempo de escape del ápice al ramoneo bovino. El modelo estadístico fue significativo, indicando que el tiempo de escape del ápice al ramoneo es diferente entre categorías de altura ( $\mathrm{F}=15,45 ; P \leq 0,001$ cuadro 1$)$ y cargas ganaderas generales $(\mathrm{F}=5,58 ; P=0,030)$. La prueba pos-hoc reveló que al año 2011, los ápices de la categoría de renovales chicos necesitan aún 20 años más de crecimiento medio para escapar al ramoneo bovino, valor significativamente superior a los renovales medianos y grandes, que al año 2011 ya lograron escapar al ramoneo o necesitan como máximo un año más de crecimiento ( $\mathrm{F}=20,39$; $P \leq 0,001$; cuadro 1)
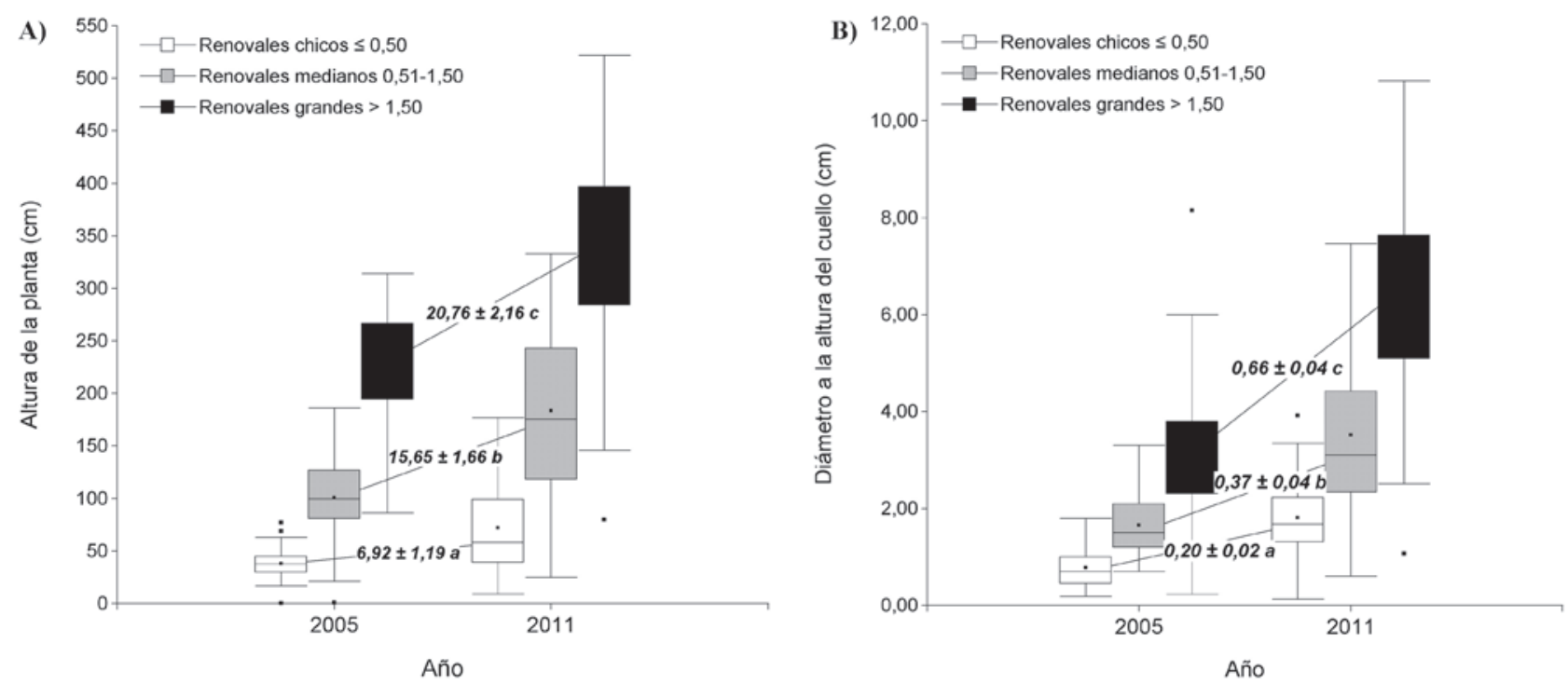

Figura 3. A) Altura media de cada categoría de altura de planta ( $\left.\pm \mathrm{EE}^{1}\right)$ de todas las parcelas para el año 2005 y 2011, respectivamente. Los conectores indican la tasa anual de crecimiento de las plantas en altura [cm año ${ }^{-1}$ ] para cada categoría de renovales las cuales difirieron significativamente entre ellas, estando esto indicado con letras diferentes. B) DAC medio de cada categoría de planta $\left( \pm \mathrm{EE}^{1}\right)$ de todas las parcelas para el año 2005 y 2011, respectivamente. Los conectores indican las tasa anual de crecimiento anual de las plantas en diámetro $[\mathrm{cm}$ año-1 $]$ para cada categoría de renovales, las cuales difirieron significativamente entre ellas, estando esto indicado con letras diferentes. ${ }^{1}$ Error Estándar.

A) Mean plant category height $\left( \pm \mathrm{SE}^{2}\right)$ from all plots for the year 2005 and 2011, respectively. Connectors -in different letters- indicate annual plant growing rates in height [ $\left.\mathrm{cm}_{\text {year }}{ }^{-1}\right]$ for each sapling category; which differed among them. B) Mean plant category diameter at the base of the trunk $\left( \pm \mathrm{SE}^{2}\right)$ from all plots for the year 2005 and 2011, respectively. Connectors indicate annual plant growing rates in diameter [cm year $\left.{ }^{-1}\right]$ for each sapling category which differs among them and it is indicated with different letters. ${ }^{1}$ Standard error. 
Número de ramificaciones. El número de ramificaciones de los renovales tuvo un aumento significativo entre el año 2005 y el año 2011 ( F = 177,37; $P \leq 0,001)$. Asimismo, se encontró una interacción significativa entre carga ganadera y año ( $\mathrm{F}=5,409 ; P=0,021$; cuadro 1$)$, haciéndose evidente que a lo largo del período de estudio hubo un mayor incremento en el número de ramificaciones de los predios de carga ganadera baja respecto a los de alta (figura 4A). $\mathrm{La}$ carga ganadera tuvo efecto marginalmente significativo sobre el números de ramificaciones $(\mathrm{F}=3,452 ; P=0,065$; figura 4A), evidenciando una tendencia de los renovales sometidos a altas cargas ganaderas a tener mayor número de ramificaciones que los de baja carga ganadera (figura 4A).

El número de ramificaciones de los renovales tuvo un incremento entre el año 2005 y el año 2011 en todas las categorías de renovales $(\mathrm{F}=171,947 ; P \leq 0,001$; figura 4B). Asimismo, se encontró una interacción significativa entre categoría y año ( $\mathrm{F}=7,604 ; P=0,001$; cuadro 1$)$, haciéndose evidente que las categorías de renovales medianos y grandes aumentaron más el número de ramificaciones respecto a los renovales chicos (figura 4B). Además, la categoría de altura de renovales tuvo un efecto significativo sobre el número de ramificaciones $(\mathrm{F}=68,753$; $P \leq 0,001$ ), siendo esta mayor en las categorías de renovales grandes respecto a los medianos, y estos a su vez mayores que los chicos (figura 4B).

Deformaciones. El efecto año sobre el índice de arbustización no fue significativo $(\mathrm{F}=0,265 ; P=0,607)$. Tampoco hubo interacción significativa entre carga y año $(F=2,799$;
$P=0,096)$, lo cual indicaría tendencias similares en ambos tratamientos para el índice de arbustización (figura 5). Sin embargo, el factor carga fue significativo $(\mathrm{F}=12,47$; $P=0,001)$, lo que reveló que una alta carga ganadera tiende a arbustizar mas los renovales de ñire respecto a carga baja.

\section{DISCUSIÓN}

Los niveles de daño por ramoneo bovino en ramas laterales de la regeneración de ñire fueron similares en establecimientos con alta carga ganadera respecto a los de baja carga ganadera general. Es posible observar una tendencia al incremento en el nivel de daño lateral a lo largo del tiempo. El daño lateral encontrado en las plantas muestreadas refleja un efecto acumulativo del ramoneo en un período de tiempo que excede el considerado en el presente estudio, y posiblemente impide encontrar diferencias entre los diferentes niveles de carga ganadera. Diversos estudios de respuesta de especies leñosas nativas a la herbivoría doméstica, hacen referencia al efecto previo y acumulado de la carga ganadera que permiten en algunos casos explicar patrones (Giorgis et al. 2010), mientras que en otros pueden confundir las respuestas de las plantas a la herbivoría (Teich et al. 2005). La prolongada temporalidad que refleja el daño lateral sobre la regeneración se aprecia de manera homogénea en toda la unidad de manejo ganadero. Sin embargo, se ha comprobado que el aumento en la presión de herbivoría en sistemas silvopastoriles de ñire como consecuencia de la disminución anual en la disponibilidad forrajera, se refleja directamente y en el mismo año en un mayor nivel de daño
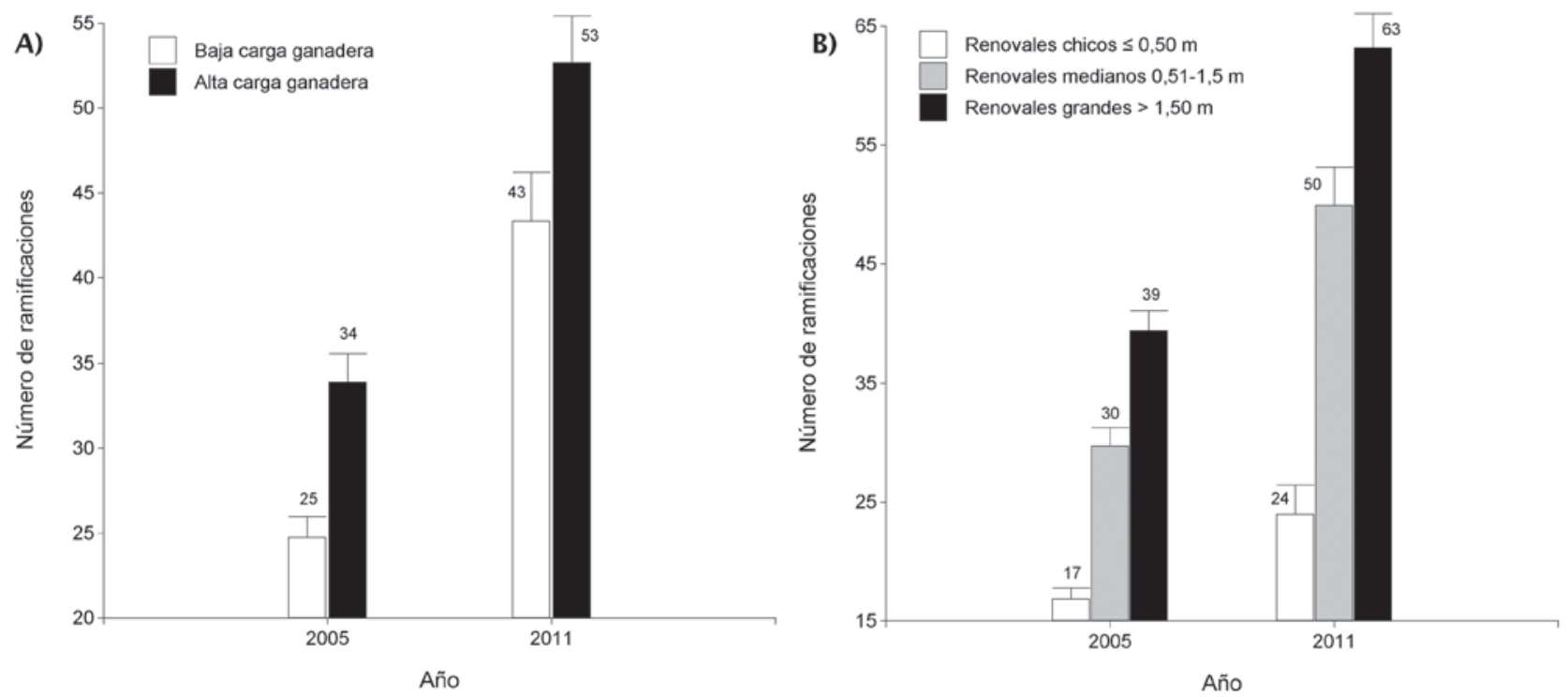

Figura 4. A) Número de ramificaciones de las plantas $(\% \pm E E)$ sometidas a baja y alta carga ganadera general para los años 2005 y 2011, respectivamente. B) Número de ramificaciones de las plantas $( \pm \mathrm{EE})$ en diferentes categorías de altura de renovales $(\leq 0,5 \mathrm{~m}$, 0,5-1,5 m y > 1,5 m) para los años 2005 y 2011, respectivamente.

A) Number of stems per plants ( \pm SE) at low and high stocking rates for sampling years, 2005 and 2011, respectively. B) Number of stems per plants $(\% \pm \mathrm{SE})$ in different plant height categories $(\leq 0.5 \mathrm{~m}, 0.5-1.5 \mathrm{~m}$ and $>1.5 \mathrm{~m})$ for both sampling years 2005 and 2011 , respectively. 


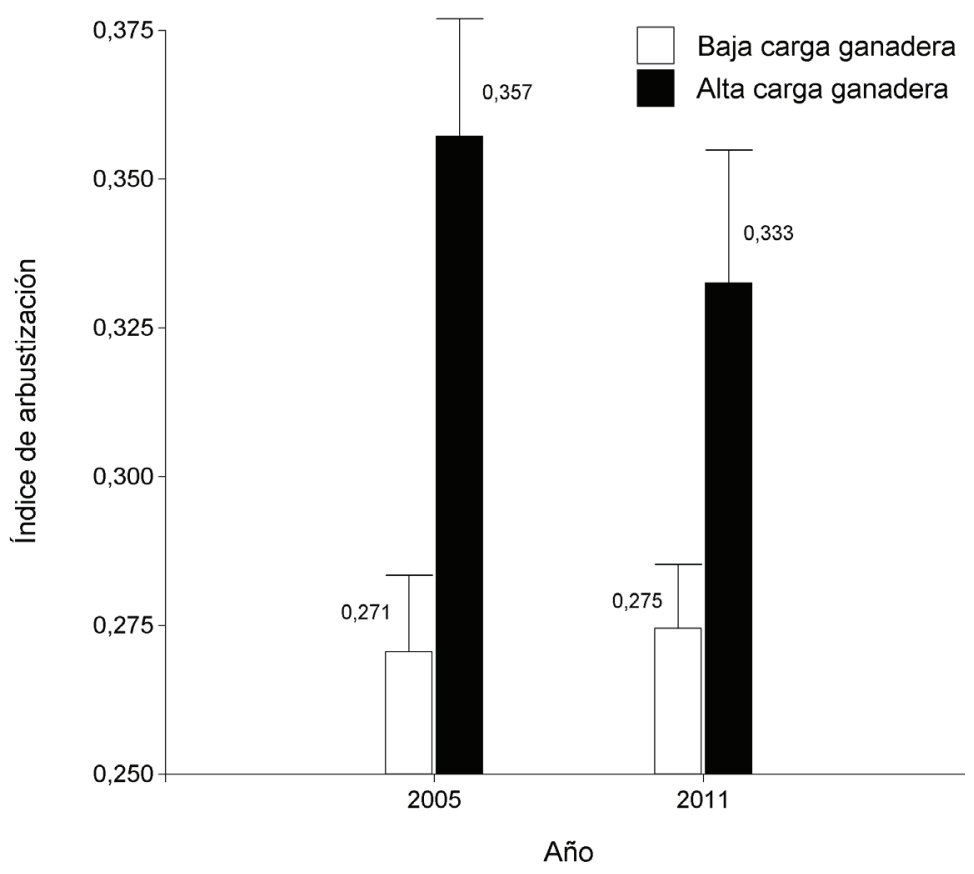

Figura 5. Valores medios de índice de arbustización de los renovales de ñire $( \pm \mathrm{EE})$ en establecimientos con baja y alta carga ganadera general para los años 2005 y 2011, respectivamente.

Mean values shrubbiness index ( \pm SE) in low and high stocking rate settlements for both sampling years 2005 and 2011, respectively.

sobre las ramas laterales (Hansen et al. 2008). De ambos resultados se deprende la necesidad de evaluar no solo niveles de daño lateral, sino también diferenciar si el daño fue realizado en la última temporada de pastoreo, o en temporadas previas. Al considerar las diferentes categorías de altura de renovales en relación al daño lateral, se puede apreciar que el ganado bovino afecta principalmente a renovales chicos y medianos (menores a 1,5 m de altura), en los que la totalidad de la planta se encuentra accesible al ramoneo. Los renovales más grandes logran disponer de ramas fuera del alcance del ramoneo, disminuyendo de esta manera el nivel de daño lateral. Esto puede explicar la mayor tasa de mortalidad de renovales chicos y más sometidos por el ramoneo, respecto a las otras categorías de mayor altura. Diversos estudios a nivel global revisados por Asner et al. (2004) han demostrado que el ramoneo ganadero reiterado compromete la regeneración natural del bosque nativo en cantidad y calidad. En algunas especies palatables de bosque nativo se encontró que los individuos al ser ramoneados intensamente en sus ramas laterales, disponen de mecanismos que le permiten tolerar el efecto del herbívoro en el corto plazo (Giorgis et al. 2010). Sin embargo, si se consideran escalas temporales o espaciales mayores, existen tendencias al retroceso de los bosques nativos cuando las cargas de herbívoros son altas respecto a la capacidad de resiliencia del bosques nativo (Cingolani et al. 2008). Por este motivo es necesario realizar ajustes adecuados de la carga ganadera bovina, impidiendo de esta manera superar los umbrales de tolerancia al daño por ramoneo (von Müller et al. 2013b).
La magnitud del daño ocasionado por el ganado bovino sobre el ápice de los renovales de ñire está determinada por la altura de las plantas y por la carga ganadera. Las plantas con alturas mayores a 1,6 m logran quedar fuera del alcance del ramoneo bovino, cuando las cargas ganaderas son moderadas a bajas. El daño provocado en el ápice tiene una temporalidad y patrón espacial diferente al del daño lateral, y es posible explicarlo con la carga bovina localizada (von Müller et al. 2012). La carga localizada permite apreciar la selectividad espacial que realiza el ganado bovino sobre determinadas áreas o rodales dentro de la unidad de manejo ganadero (Quinteros et al. 2012, von Müller et al. 2013a). El ganado bovino tiene preferencia en su dieta por el género Nothofagus (Vila y Borrelli 2011, Quinteros et al. 2013), y por este motivo, las plantas de ñire van a ser ramoneadas indefectiblemente. Sin embargo, si las cargas ganaderas son bajas, la magnitud del daño apical sobre la regeneración va a estar localizada en determinados sitios preferidos dentro de la unidad de manejo (Quinteros et al. 2012). Por el contrario, si las cargas ganaderas son altas, el daño apical va a ser de magnitud en toda la unidad de manejo ganadero, sin posibilidad de que algunos renovales escapen al ramoneo para garantizar la regeneración. En estudios previos en bosque de ñire se encontró que la altura de ramoneo del ápice osciló entre 1,5 y 2,5 m de altura (Hansen et al. 2008, Peri et al. 2009). Con altas cargas ganaderas, el ganado presiona los renovales de ñire inclinando las plantas y accediendo tanto a ramas laterales fuera del alcance de su boca como a su ápice 
(observación personal del autor). Los renovales de ñire de categorías grandes logran diámetros a la altura del cuello mayores, lo cual se ve reflejado en una mayor estabilidad frente a la presión del ganado aún en condiciones de altas cargas ganaderas (Hansen et al. 2004). Al ser ramoneado el ápice, los renuevos disminuyen su tasa de crecimiento en altura y esto se traduce en más tiempo para llegar a la altura de escape al ramoneo. En el largo plazo, el ramoneo reiterado del ápice va debilitando los renovales, afectando la supervivencia y la calidad de plantas (Briske 1996). Estos efectos negativos pueden provocar retrocesos irreversibles de la masa boscosa, relegándola a zonas protegidas de los herbívoros (Asner et al. 2004).

Los renovales de ñire continuaron creciendo en altura y diámetro a lo largo del período de estudio en todas las categorías a pesar del ramoneo bovino permanente. Sin embargo, las tasas anuales de crecimiento de los renovales grandes, menos sometidos por el ramoneo, fue mucho mayor que los medianos y chicos. En una primera interpretación, sería posible inferir que las diferencias en las tasas de crecimiento pueden atribuirse a la posición de las categorías por su edad en la curva de crecimiento (Ivancich 2013). Sin embargo, por la altura de lo individuos dominantes de los rodales es posible inferir que las parcelas de muestreo en encuentran en una clase de sitio intermedia/buena (Clase II, según Ivancich et al. 2011). En esta clase de sitio forestal, según los modelos de Ivancich (2013), las curvas de crecimiento exponencial de los renovales llegan hasta aproximadamente los 5 años de edad. Por los diámetros a la altura del cuello medidos inicialmente en 2005, los renovales considerados en el presente estudio tendrían en todos las categorías al menos cinco años de edad al inicio del estudio, y por ende estarían en su etapa de crecimiento lineal (Ivancich et al. en prensa). Por este motivo, las diferencias en las tasa de crecimiento son debidas a que los renovales chicos, más sometidos por el ramoneo permanente, no pueden expresar su potencial de crecimiento. Por el contrario, los renovales grandes que escapan al ramoneo del ápice, compensan la biomasa removida en altura y diámetro, y logran recuperarse del efecto de la herbivoría doméstica. Sobre la base de estos resultados, es posible inferir que el ñire responde al ramoneo bovino, compensando en las ramas superiores y en altura, la biomasa removida en las ramas inferiores (Belsky et al. 1993). Asimimo, el crecimiento compesatorio que expresan los renovales de mayor tamaño define incrementos también en los diámetros a la altura del cuello, logrando de esta manera plantas más estables frente a la herbivoría. Este mecanismo de crecimiento compensatorio ha sido demostrado en otras especies leñosas arbóreas con alta palatabilidad y sometidas a cargas ganaderas bajas a moderadas (Giorgis et al. 2010). Sin embargo, cuando las cargas ganaderas son excesivamente altas, el crecimiento compensatorio se ve interrumpido y las plantas no logran recuperarse del ramoneo (Belsky et al. 1993). La mayor tasa de crecimiento de los renovales medianos a grandes, y su mayor altura media, permite que el tiempo de escape del ápice al ramoneo sea independiente de la carga ganadera. Por el contrario, los renovales de ñire chicos, al tener menores tasas de crecimiento y estar más accesibles al ganado bovino, necesitan alrededor de 20 años en condiciones de pastoreo para que el ápice logre escapar del ramoneo. En condiciones de alta carga ganadera, la susceptibilidad al ramoneo del ápice aumenta aún más, creando de esta manera una retroalimentación positiva que puede comprometer a la regeneración de ñire si no se logran individuos que logren escapar al ramoneo.

Los renovales incrementaron el número de ramificaciones a lo largo del período de estudio independientemente del efecto del ramoneo bovino. Las plantas de ñire al tolerar la herbivoría, continúan con su crecimiento y los fotosintatos son destinados a nuevas estructuras aéreas (Adler et al. 2004). Los renovales sometidos a cargas altas ganaderas tuvieron en todos los casos un mayor número de ramificaciones respecto a los de cargas bajas. En renovales chicos el ramoneo del ápice provoca la ruptura de la dominancia apical y determina que las yemas subyacentes crezcan y se desarrollen, generando mayor número de ramificaciones (Belsky 1986). En renovales grandes, cuando el ápice escapa al ramoneo, se desencadena un crecimiento vigoroso en altura con desarrollo de gran cantidad de ramificaciones secundarias. El incremento en el número de ramificaciones es otra adaptación, sustentada en el crecimiento compensatorio, que confirma la tolerancia del ñire a la herbivoría (Belsky et al. 1993, Briske 1996). Por otro lado, el índice de arbustización es considerado un indicador de calidad de regeneración de especies leñosas, cuanto mayor es el valor, menor es la calidad y viceversa (Renison et al. 2005). La morfología de la regeneración de ñire en predios con alta carga ganadera es más arbustizada respecto a los de bajas carga ganadera condicionando la calidad de la regeneración. La arquitectura de las plantas refleja el efecto de la herbivoría en un período prolongado de tiempo (Briske 1996, Asner et al. 2004), y por este motivo que no fue posible encontrar cambios durante el tiempo de estudio.

\section{CONCLUSIONES}

La adecuación de la carga ganadera y el monitoreo de la altura de la regeneración establecida son aspectos relevantes a tener en cuenta para la sustentabilidad de los sistemas silvopastoriles en bosque de ñire. El daño apical de la regeneración de ñire es un indicador clave que define la altura de la regeneración establecida y debe ser tomado en cuenta cuando se planifique el manejo ganadero ya que afecta la sobrevivencia y la calidad futura de las plantas.

Los establecimientos manejados con cargas ganaderas bajas durante la veranada, por debajo de 0,4 UG ha-1, deberán lograr renovales de al menos 1,6 m de altura para garantizar la renovación de la masa boscosa. Este manejo permite que gran parte de la regeneración establecida logre 
escapar al efecto del ramoneo bovino, y luego desarrollar mecanismos de crecimiento compensatorio de $21 \mathrm{~cm}$ año ${ }^{-1}$ en altura y $0,7 \mathrm{~cm}$ año ${ }^{-1}$ en diámetro que le permiten al ñire tolerar la herbivoría doméstica. Bajo estas condiciones es posible encontrar una compatibilidad entre ganadería y conservación del bosque de ñire.

Por el contrario, establecimientos manejados por un tiempo prolongado con cargas ganaderas altas, por encima de $0,8 \mathrm{UG} \mathrm{ha}^{-1}$, determinan que gran parte de la regeneración del bosque de ñire se encuentre sometida por el ramoneo, con plantas en promedio más bajas y evidentes signos de arbustización. Estas condiciones dejan además el ápice de los renovales más accesible a los herbívoros domésticos y determinan tasas de crecimiento de $7 \mathrm{~cm}$ año ${ }^{-1}$ en altura y de $0,2 \mathrm{~cm}$ año ${ }^{-1}$ en diámetro. Para recuperar la regeneración de ñire bajo estas condiciones es necesario realizar exclusiones ganaderas de aproximadamente 20 años para permitir recuperar la regeneración establecida. Este manejo compromete a la regeneración de ñire en calidad y cantidad, y por lo tanto al bosque futuro.

\section{REFERENCIAS}

Asner G, A Elmore, L Olander, R Martin, A Harris. 2004. Grazing systems, ecosystem responses, and global change. Annual Review on Environmental Resources 29: 261-299.

Bahamonde HA, PL Peri, LH Monelos, G Martínez Pastur. 2011. Aspectos ecológicos de la regeneración por semillas en bosques nativos de Nothofagus antarctica en Patagonia Sur, Argentina. Bosque 32(1): 20-29.

Belsky AJ. 1986. Does herbivory benefit plants? A review of the evidence. American Naturalist 127: 870-892.

Belsky AJ, WP Carson, CL Jensen, CA Fox. 1993. Overcompensation by plants: herbivore optimization or red herring? Evolutionary Ecology 7: 109-21.

Blackhall M, E Raffaele, T Veblen. 2008. Cattle affect early postfire regeneration in Nothofagus dombeyi-Austrocedrus chilensis mixed forest in northern Patagonia, Argentina. Biological conservation 141(9): 2251-2261.

Briske DD. 1996. Strategies of plant survival in grazed systems: a functional interpretation. In Hodgson J, AW Illius eds. The ecology and management of grazing systems. Wallingford, UK. CAB International. p. 37-67.

Cingolani AM, D Renison, PA Tecco, DE Gurvich, M Cabido. 2008. Predicting cover types in a mountain range with long evolutionary grazing history: a GIS approach. Journal of Biogeography 35: 538-551.

Donoso Z C, L Steinke, A Premoli. 2006. Nothofagus antarctica (G. Forster) Oerst. Ñirre, Ñire, Ñiré, Anís (Tierra del Fuego) Ñirre: de Ngërü (mapudungun): zorro. In Donoso Zegers $\mathrm{C}$ ed. Las especies arbóreas de los bosques templados de Chile y Argentina. Autoecología. Valdivia, Chile. Marisa Cuneo. p 401-410.

Fernández PE. 2008. Taphonomy and zooarchaeology in the Neotropics: A view from northwestern Patagonian forest and steppe. Quaternary International 180: 63-74.

Giorgis M, A Cingolani, I Teich, D Renison, I Hensen. 2010. Do Polylepis australis trees tolerate herbivory? Seasonal patterns of shoot growth and its consumption by livestock.
Plant Ecology 207: 307-319.

Guitart E. 2004. Diagnóstico productivo orientado a establecer la capacidad del sector ganadero del NO del Chubut para involucrarse y sostener un proyecto de diferenciación por calidad del producto carne vacuna. Esquel, Argentina. INTA. $48 \mathrm{p}$.

Hansen N, L Tejera, M Fertig. 2004. Módulo 2. Desarrollo de sistemas silvopastoriles en bosques de Nothofagus antarctica. In Alternativas de manejo sustentable para el manejo forestal integral de los bosques de la Patagonia. Capítulo 3. Sistemas silvopastoriles en Chubut. Informe PIARFON p. 671-680.

Hansen N, M Fertig, M Escalona, L Tejera, W Opazo. 2008. EcoNothofagus. Segunda Reunión sobre Nothofagus en la Patagonia. Ramoneo en regeneración de ñire y disponibilidad forrajera. Esquel. Chubut, Argentina. p. 137-142.

Hansen NE, von Müller A, CE Lloyd. 2014. Disponibilidad forrajera en el Bosque Andino Patagónico. Cartilla Técnica INTA EEAf Esquel. Sistemas Silvopastoriles. p. 215-218. [http://inta.gob.ar/documentos/disponibilidad-forrajera].

Hofmann RR. 1989. Evolutionary steps of ecophysiological adaptation and diversification of ruminants: a comparative view of their digestive system. Oecología 78: 443-457.

Ivancich H, G Martínez Pastur, PL Peri. 2011. Modelos forzados y no forzados para el cálculo del índice de sitio en bosques de Nothofagus antarctica en Patagonia Sur. Bosque 32(2): 135-145.

Ivancich HS. 2013. Relaciones entre la estructura forestal y el crecimiento del bosque de Nothofagus antarctica en gradientes de edad y calidad de sitio. Tesis Doctoral. Universidad Nacional de La Plata. Argentina. p 180.

Ivancich H, G Martínez Pastur, MV Lencinas, JM Cellini, PL Peri. In press. Proposals for Nothofagus antarctica diameter growth estimation: simple vs. global models. Journal of Forest Science 60 (7).

Marquardt S, A Marquez, H Bouillot, S Beck, A Mayer, M Kreuzer, y otros. 2009. Intensity of browsing on trees and shrubs under experimental variation of cattle stocking densities in southern Bolivia. Forest Ecology and Management 258(7): 1422-1428.

Oesterheld M, OE Sala, SJ McNaughton S. J. 1992. Effect of animal husbandry on herbivore-carrying capacity at a regional scale. Nature 356: 234-6.

Panigatti JL. 2010. Argentina 200 años, 200 suelos. Buenos Aires, Argentina. INTA. 345 p.

Peri P, S Ormaechea, L Huertas. 2009. Protección de renovales de ñire. Producción Forestal 4: 15-16.

Premoli A. 1991. Morfología y capacidad germinativa en poblaciones de Nothofagus antarctica (G. Forster) Oerst. del noroeste andino-patagónico. Bosque 12(2): 53-59.

Quinteros P, N Hansen, A Kutschker. 2010. Composición del sotobosque de ñire (Nothofagus antarctica) en función de la estructura del bosque. Ecología Austral 20: 225-234.

Quinteros CP, PM López Bernal, ME Gobbi, JO Bava. 2012. Distance to flood meadows as a predictor of use of Nothofagus pumilio forest by livestock and resulting impact, in Patagonia, Argentina. Agroforestry Systems 84: 261-272.

Quinteros CP, PM López Bernal, SM Feijoó, JO Bava, NS Arias. 2013. Dieta de verano de bovinos pastoreando en bosques de lenga (Nothofagus pumilio) y mallines de Chubut, Argentina. Revista FCA UNCuyo 45(1): 285-292. 
Raffaele E, T Kitzberger, T Veblen. 2007. Interactive effects of introduced herbivores and post-flowering die-off of bamboos in Patagonian Nothofagus forest. Journal of Vegetation Science 18: 371-378.

Renison D, AM Cingolani, R Suarez, E Menoyo, C Coutsiers, A Sobral, I Hensen. 2005. The Restoration of Degraded Mountain Woodlans: Effects of Seed Provenance and Microsite Characteristics on Polylepis australis Seedling Survival and Growth in Central Argentina. Restoration Ecology 13: 129-135.

Reque J A, M Sarasola, J Gyende, ME Fernández. 2007. Caracterización silvícola de ñirantales del norte de la Patagonia para la gestión forestal sostenible. Bosque 28(1): 33-45.

Teich I, AM Cingolani, D Renison, I Hensen, M Giorgis. 2005. Do domestic herbivores retard Polylepis australis Bitt. woodland recovery in the mountains of Córdoba, Argentina? Forest Ecology and Management 219: 229-241.

Van Soest, PJ. 1996. Allometry and Ecology of Feeding Behavior and Digestive Capacity in Herbivores: A Review. Zoo Bio- logy 15: 455-479.

Vila AR, L Borrelli. 2011. Cattle in the Patagonian forests: Feeding ecology in Los Alerces National Reserve. Forest Ecology and Management 261(7): 1306-1314.

von Müller AR, AM Cingolani, MV Vaieretti, D Renison. 2012. Estimación de carga bovina localizada a partir de frecuencia de deposiciones en un pastizal de montaña. Ecología Austral 22: 178-187.

von Müller AR, C Lloyd, N Hansen, V Nakamatsu. 2013a. Selectividad bovina en bosque de ñire: influencia de factores tróficos, abióticos y de manejo. In VI Congreso Nacional de Pastizales y III del Mercosur. Santa Rosa, La Pampa, Argentina.

von Müller AR, C Lloyd, N Hansen, CG Buduba, G Ciari. 2013b. Adecuación de la carga ganadera para asegurar la conservación de la estructura y los servicios ecosistémicos en el bosque andino patagónico. Jornadas II Jornadas Forestales Patagónicas y $2^{\circ}$ Congreso Internacional Agroforestal Patagónico. El Calafate, Santa Cruz, Argentina.

Recibido: 09.04.14

Aceptado: 04.09.14 